\title{
Study of real-time biomimetic CPG on FPGA: behavior and evolution
}

\author{
Timothée Levi $^{* 1}$, Yanchen Guo ${ }^{1}$, Kazuyuki Aihara ${ }^{1}$, Takashi Kohno ${ }^{1}$ \\ IIS * The University of Tokyo, 4-6-1 Komaba, Meguro-ku \\ Tokyo, 153-8505, Japan ${ }^{1}$
}

E-mail:_evi@sat.t.u-tokyo.ac.jp,guoyanchen@sat.t.u-tokyo.ac.jpaihara@sat.t.u-tokyo.ac.jp,kohno@sat.t.u-tokyo.ac.jp

\begin{abstract}
Locomotion is one of the most basic abilities in animals. Neurobiologists have established that locomotion results from the activity of half-center oscillators that provides alternation of bursts. Central Pattern Generators (CPGs) are neural networks capable of producing rhythmic patterned outputs without rhythmic sensory or central input. We propose a network of several biomimetic CPGs using biomimetic neuron model and synaptic plasticity. This network is implemented on a FPGA (Field Programmable Gate Array). The network implementation architecture operates on a single computation core and in real-time. The real-time implementation of this CPGs network is validated by comparing it with biological data of leech heartbeat neural network. From these biomimetic CPGs, we use them for robotic applications and also for biomedical research to restore lost synaptic connections.
\end{abstract}

Keywords: Biomimetic neural network, CPG, FPGA, Silicon neuron

\section{Introduction}

Locomotion in robotic and neuroscience field is highly important, and its complexity depends on the degree of fitting the nature. The locomotion is based on the activity of half-center oscillators. The first half-center oscillator was proposed by Brown ${ }^{1}$ in 1914. These oscillators which mimic the rhythmic movements are programmed by central pattern-generating (CPG) networks consisting of neural oscillators ${ }^{2}$. These CPGs provides alternating burst without rhythmic sensory or central input ${ }^{3}$.

Usually, in robotic field, the CPGs are made using simple neuron-models, and are not in biological time scale (accelerated time). These two specifications do not able bio-hybrid experiments between biological cells and artificial cells. Therefore, we could say that these CPGs are bio-inspired and not biomimetic. A bio-inspired system is inspired by nature for conducting engineering task (neural network for image processing, data mining, etc.). A biomimetic system mimics the nature for simulating the living behavior and/or replaces it (rebuilding the living part). Biomimetic system can perform bio-hybrid experiments. While multi-legged robots need CPG to move or coordinate their movements, researchers implement an Amari-Hopfield $\mathrm{CPG}^{4}$ or basic CPGs like Van der $\mathrm{Pol}^{5}$, modeled as non-linear oscillators. Those models provide sinusoidal oscillations that are not biomimetic.

To be closer to the nature, we developed biomimetic CPGs. These CPGs will mimic the animal locomotion like swimming in xenopus, salamander ${ }^{6}$, and lamprey ${ }^{7}$, as well as leech heartbeat system $^{8,9}$. This article is decomposed into three parts: description and results of the biomimetic network of CPGs, applications on robotic field and biomedical neuroscience research. 


\section{Biomimetic CPGs}

We propose a network of hundreds biomimetic CPGs using biomimetic neuron, synapse and short-term plasticity models. For instance the salamander locomotion needs $40 \mathrm{CPGs}^{6}$.

\subsection{Model of the Leech Heartbeat system}

Our CPG is based on the Leech heartbeat neural network system ${ }^{9}$. The simplest biomimetic CPG is the one responsible for the leech heartbeat (Figure 1). This CPG consists of two neurons, connected to each other via inhibitory synapses. As this is the most basic network, it is ideal for achieving a better understanding of various phenomena that control its activity. This pair of neurons, also known as an elementary oscillator, is also the smallest network capable of generating oscillations under normal conditions. We add two excitatory neurons connected with excitatory synapses for detecting burst activity of the elementary oscillator.

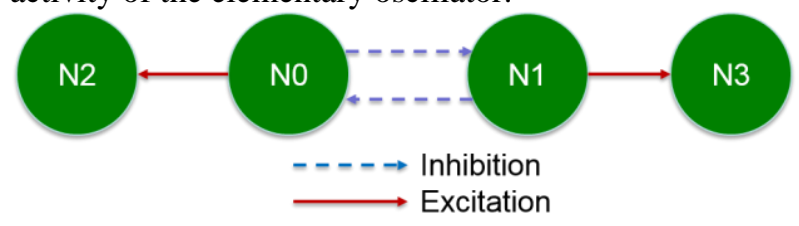

Fig. 1. A diagram of the CPG in the leech heartbeat system, composed of one elemental oscillator (N0 and N1). Synapses between N0-N1 couple are inhibitory. N2 and $\mathrm{N} 3$ are burst-detector neurons. They are spiking just one time when a burst occurs in their connected neuron. N2$\mathrm{N} 3$ are respectively connected to N0-N1 by excitatory synapses.

\subsection{Neuron, synapse and short-term plasticity model}

The biomimetic CPG that we designed is presented in details in ${ }^{10,11}$. No CPGs network and no real-time period modifications were possible in ${ }^{10}$.

The neuron model choice is very important, because we have to choose between the biological plausibility and the cost computation. The choice of model was based on two criteria: the family of neurons able to be reproduced and the number of equations. These criteria were used to compare several models, from the simple one: the Leaky Integrate and Fire model (LIF) ${ }^{12}$ to the most complex one: the Hodgkin-Huxley model $(\mathrm{HH})^{13}$.

In the article by Hill ${ }^{9}$, $\mathrm{HH}$ model is used to reproduce the leech heartbeat system. Nevertheless, the HH model requires a large number of parameters and the LIF model is too simple for reproducing the neural network behavior in Hill'.

The Izhikevich model (IZH) ${ }^{14}$ represents a good trade-off as it is based on two equations and is capable of reproducing many different families of neurons by changing four parameters. Furthermore, this model is resource-frugal, a key advantage when the aim is to design a large CPG network embedded in the same board. As we chose the neuron model, we have to choose carefully the synapse model. This model should be biomimetic with few resources. In case of excitatory (inhibitory) synapse, a positive contribution (negative contribution) is added to the postsynaptic neuron when the presynaptic neuron spikes. Furthermore, we add the short-term plasticity described by Izhikevich ${ }^{15}$ that is different from Spike-Timing Dependent Plasticity (STDP) and that manages the depression or the facilitation of the synaptic strength. This synaptic plasticity is similar to the one described in Hill ${ }^{9}$.

\subsection{Design of real-time system}

This network is implemented in a digital platform: Xilinx Kintex-7 FPGA (Field Programmable Gate Array). The network implementation architecture operates on a single computation core. This digital system works in real-time, requires few resources and is low power consumption. It is essential in robotic applications. The implementation of one CPG is validated by comparing it with biological data of leech heartbeat neural network ${ }^{10}$. These CPGs could reproduce biological activity of CPG neural networks not only in terms of period but also in terms of variation of duty cycle and bursting period.

This implementation is low resources. Table 1 presents the used resources for 128 CPGs in Xilinx Kintex-7 FPGA family. Using full resources, we can implement 984 CPGs. For instance, salamander locomotion needs 40 CPGs.

Table 1. Utilization of the resources for the implementation of $128 \mathrm{CPGs}$

\begin{tabular}{|l|c|c|c|}
\hline Kintex-7 & Utilization & Available & Percentage \\
\hline LUT & 26795 & 203800 & $\mathbf{1 3 , 1}$ \\
\hline FF & 9479 & 407600 & $\mathbf{2 , 3}$ \\
\hline BRAM & 43 & 445 & $\mathbf{9 , 6}$ \\
\hline DSP & 40 & 840 & $\mathbf{4 , 7}$ \\
\hline
\end{tabular}




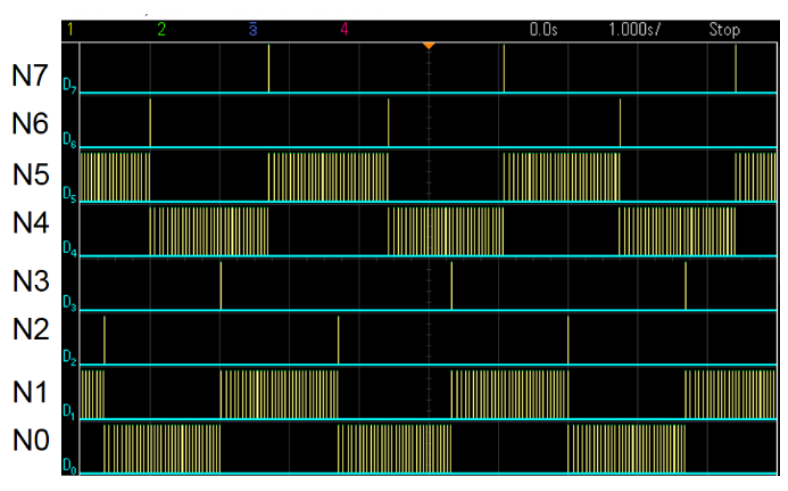

Fig. 2. Two real-time CPGs (N0-N1 and N4-N5) with their burst-detection neurons (N2-N3 and N6-N7). Time axis is one second by division.

Figure 2 describes the FPGA implementation results for two CPGs (N0-N1 and N4-N5) with their two burstdetector neurons (N2-N3 and N6-N7).

The real-time behavior of CPG system is mandatory as we can use for robotic applications (section 3), biohybrid experiments but also to simulate different topologies and lost connections as it can exist in the nature (section 4). Using software, simulation time is too long as neuron model and synaptic plasticity use complex non-linear differential equations. Thanks to this real-time system, we can simulate several topologies of CPG network and then find some treatment protocol when neuronal connections are lost.

\section{Robotic applications}

Usually simple CPGs are often used for robotic application but biomimetic CPGs are rarely used. Nevertheless some few works ${ }^{6,16-20}$ developed biomimetic motion for robotics. To demonstrate the feasibility of using our biomimetic CPGs. We create forward locomotion for artificial salamander with different periods and different phases between CPGs. We reproduce the forward locomotion explained in ${ }^{16}$ using a loop of seven CPGs (from CPG0 to CPG6) with crossed inhibition connections between them. Figure 3 describes two different forward locomotions with different periods. We can modify the period in real-time by tuning the weight of the crossed synaptic connections between the CPGs. We also investigated a master CPG which controls the activity of seven CPGs with excitatory connections between the master CPG and CPG0. Thanks to this master CPG, we can directly choose the frequency and phases between CPGs using just two parameters (period of the master CPG and value of the excitatory synapse weight).
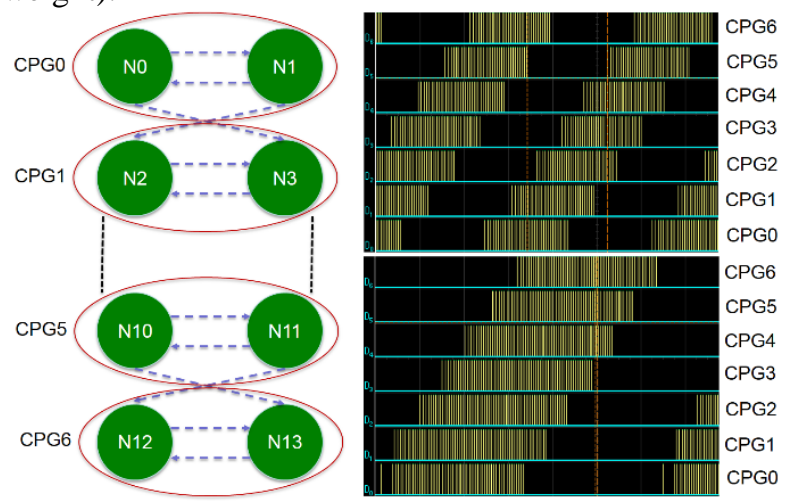

Fig. 3. Forward locomotion with two examples of speed using a chain of 7 CPGs. Time axis is one second by division. Signal are the left neuron (N0, N2, N4, N6, N8, N10 and N12) of each CPG.

\section{Biomedical applications}

How to restore locomotion activity when some connections are lost? We simulated several cases. Here, we present one study where one CPG does not work anymore as the inhibition connection between N6 and N7 is lost. In that case, N7 is always firing and N6 is always inhibited as Figure 4 (top) describes it.

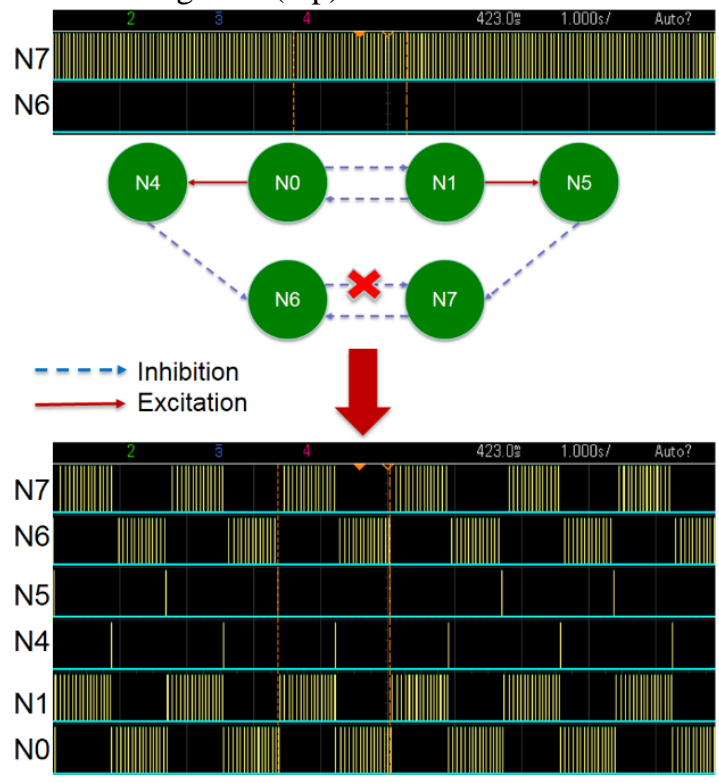

Fig. 4. Reconstruction of a lost synaptic connection. The couple N6-N7 recovers its CPG activity. Time axis is one second by division. 
This result shows that thanks to new synaptic connections from other CPG, we can restore a lost activity (figure 4 bottom). As our biomimetic CPGs can be connected to living neurons, this research can be extent to bio-hybrid experiments and uses these biomimetic CPGs as neuroprostheses during spinal cord lesion.

\section{Conclusion}

This article demonstrates a real-time network of biomimetic CPGs. This system can be used in robotic applications for smoother and closer behavior to biological animals. It can also be used for bio-hybrid robotic (living part and artificial part) as it was demonstrated that this system can be connected to biological neurons ${ }^{21,22}$. Another advantage is that realtime systems can simulate several topology of CPGs and simulate different hypothesis and protocol treatment for biomedical applications like spinal cord injury ${ }^{23}$.

\section{Acknowledgements}

Authors thank Social Cooperation Program for BrainMorphic AI to Resolve Social Issues, IIS, UTokyo.

\section{References}

1. T. Brown, On the nature of the fundamental activity of the nervous centres; together with an analysis of the conditioning of rhythmic activity in progression and a theory of the evolution of function in the nervous system. Journal of Physiology, 48, 18-46, 1914

2. E. Marder and D. Bucher, Central pattern generators and the control of rhythmic movements. Current Biology 11, 986-996, 2001

3. S. Hooper, Central Pattern Generators, Current Biology, 10, 176-177, 2000

4. S. Amari, Characteristic of the random nets of analog neuron-like elements, IEEE Trans. on System, Man and Cybernetics, 2, 643-657, 1972

5. B. Van Der Pol, The heartbeat considered as a relaxation oscillation, and an electrical model of the heart. Philosophical Magazine, 6, 763-775, 1928

6. A. Ijspeert, A. Crespi, D. Ryczko, J. Cabelguen, From swimming to walking with a salamander robot driven by a spinal cord model. Science, 315, 1416-1420, 2007

7. AH. Cohen, GB. Ermentrout, T. Kiemel, N. Kopel, KA. Sigvardt, TL. Williams, Modelling of intersegmental coordination in the lamprey central pattern generator for locomotion. Trends in Neuroscience. 15, 434-438, 1992

8. GS. Cymbalyuk, Q. Gaudry, MA. Masino, R.L. Calabrese, Bursting in Leech Heart Interneurons: Cell-Autonomous and Network-Based Mechanisms. J. Neuroscience, 22, 10580-10592, 2002

9. AA. Hill, J. Lu, MA. Masino, OH Olsen, RL Calabrese, A model of a segmental oscillator in the leech heartbeat neuronal network, J Comput Neurosci., 10, 281-302, 2001

10. M. Ambroise, T. Levi, S. Joucla, B. Yvert, S. Saïghi, Realtime biomimetic Central Pattern Generators in an FPGA for hybrid experiments. Frontiers in Neuroscience, 7, 215, 2013

11. M. Ambroise, T. Levi, Y. Bornat, S. Saïghi, Biorealistic spiking neural network on FPGA, 47th Annual Conference on Information Sciences and Systems CISS, 1-6, 2013

12. G. Indiveri, Synaptic plasticity and spike-based computation in VLSI networks of integrate-and-fire neurons. Neural Information Processing, Letters and Reviews, 11, 135-146, 2007

13. AL. Hodgkin, AF. Huxley, A quantitative description of membrane current and its applications to conduction and excitation in nerve, The Journal of Physiology, 117, 500544, 1952

14. E.M. Izhikevich, Simple model of spiking neurons. IEEE Transactions on Neural Networks, 14, 1569- 1572, 2003

15. E.M. Izhikevich and G. Edelman, Large-scale model of mammalian thalamocortical systems, Proceedings of the National Academy of Sciences of the United States of America, 105, 3593-3598, 2008

16. T. Matsuo, T. Yokoyama, D. Ueno, K. Ishii, Biomimetic Motion Control System Based on a CPG for an Amphibious Multi-Link Mobile Robot, Journal of Bionic Engineering, 91-97, 2008

17. J.H. Barron-Zambrano and C. Torres-Huitzil, FPGA implementation of a configurable neuromorphic CPGbased locomotion controller, Neural Netw.45, 50-61, 2013.

18. C. Li, R. Lowe, T. Ziemke, Humanoids Learning to Walk : a Natural CPG-Actor-Critic Architecture, Frontiers in Neurorobotics, 7:5, 2013

19. E. Donati, F. Corradi, C. Stefanini, G. Indiveri, A spiking implementation of the lamprey's Central Pattern Generator in neuromorphic VLSI, IEEE BioCAS 2014,2014

20. C. Qi, W.Jiang, S. Yang, Y. Qin, B. Deng, W. Xile, A realtime FPGA implementation of a biologically inspired central pattern generator network, Neurocomputing, 244, 63-80, 2017

21. S. Joucla, M. Ambroise, T. Levi, T. Lafon, P. Chauvet, S. Saighi, Y. Bornat, N. Lewis, S. Renaud, B. Yvert, Generation of locomotor-like activity in the isolated rat spinal cord using intraspinal electrical microstimulation driven by a digital neuromorphic CPG, Frontiers in neuroscience, 10, 2016

22. T. Levi et al., Biomimetic neural network for modifying biological dynamics during hybrid experiments, Journal on Artificial Life and Robotics, May 2017

23. M. Capogrosso et al., A brain-spine interface alleviating gait deficits after spinal cord injury in primates, Nature, 539, 284-288, November 2016 